\title{
Analysis of mucin composition in gastric juices of chronic rheumatic patients with upper gastrointestinal damage
}

\author{
Tomoaki Ikezawa ${ }^{1}$, Takafumi Ichikawa ${ }^{2}$, Ken Adachi ${ }^{3}$, Satoshi Sugano ${ }^{3}$, Tatsuya Ojima ${ }^{3}$, Youko Nakamura ${ }^{3}$, \\ Yukio WatANABE ${ }^{3}$ and Kazuhiko ISHIHARA ${ }^{4}$ \\ Departments of ${ }^{1}$ Internal Medicine and ${ }^{2}$ Biochemistry, Kitasato University School of Medicine, and ${ }^{4}$ Department of Biochemistry, Ki- \\ tasato University School of Allied Health Sciences, Sagamihara, Kanagawa 228-8555, and ${ }^{3}$ Department of Internal Medicine, National \\ Sagamihara Hospital, Sagamihara, Kanagawa 228-8522, Japan
}

(Received 23 May 2005; and accepted 13 June 2005)

\begin{abstract}
Assessment of the mucin subclasses in the gastric juices of severe chronic rheumatoid arthritis (RA) patients was compared with non-RA cases which received the eradication treatment of Helicobacter pylori (H. pylori). Gastric juice samples were obtained from 8 RA patients ( 5 for H. pylorinegative and 3 for $H$. pylori-positive) and 5 control subjects in which we confirmed the successful eradication of $\mathrm{H}$. pylori. The gastric luminal mucins were extracted and isolated by the ethanol precipitation method. These mucin solutions were digested with chymotrypsin, dialyzed, lyophilized, and redissolved. The obtained specimen was applied to an ion exchange column containing DEAE-Sepharose CL-6B and eluted with a discontinuous salt gradient in three salt steps. The gastric luminal mucins were divided into three fractions based on the distinctive sialic acid content. The proportion of acidic mucin rich in sialic acid from the gastric juice of RA patients without the $H$. pylori infection was significantly lower than those RA patients with $H$. pylori or the control subjects. A decrease in the acidic mucin content after eradication of $H$. pylori was commonly observed in all the control subjects. Our investigation raises the possibility that the gastric mucosae of RA patients have resistance against $H$. pylori infection. And the analysis of the composition in the gastric luminal mucin may be a very useful tool for the evaluation of gastric homeostasis in RA patients.
\end{abstract}

Helicobacter pylori (H. pylori) is now accepted as a human gastric pathogen causing chronic active gastritis, gastric and duodenal ulcers. The primary habitat of this bacterium is within the mucus gel layer of the stomach. Although the rate of $H$. pylori infection is very high throughout adulthood in Japan (3, 10 ), several trials indicate that patients with rheumatoid arthritis (RA) tend to have a low prevalence of

Address correspondence to: Dr. Takafumi Ichikawa, Department of Biochemistry, Kitasato University School of Medicine 1-15-1 Kitasato, Sagamihara, Kanagawa 228-8555, Japan.

Tel: +81-42-778-9267, Fax: +81-42-778-8441

E-mail: t.ichika@kitasato-u.ac.jp
H. pylori infection $(12,14)$. Certain anti-rheumatic gold compounds were reported to have anti- $H$. pylo$r i$ activity (8). However, few clinical trials from the viewpoint of defense for humans as a bacterial host are found in the literature.

Mucin, a major component of the mucus, is considered to be one of the principal factors in the physiological defense mechanism of the gastrointestinal mucosa (1). Considerable interest has been stimulated by the report of the selective depletion of a colonic mucin subclass in specific bowel disease (13). Although human gastric mucin has been shown to bind to $H$. pylori $(6,15)$ in in vitro studies, it is not clear how this substance is associated with the infection, particularly with regard to whether specif- 
ic alteration of the gastric luminal mucin is concerned with the $H$. pylori infection. We have already established a simple means of extraction and measurement of the mucin in human gastric juices $(4,7)$. In the present study, we have attempted an analysis of the mucin composition in the gastric juices of severe chronic RA patients compared with non-RA cases that received the eradication treatment of $H$. pylori.

\section{MATERIALS AND METHODS}

Patients. We studied 8 women patients with RA (class IV) diagnosed according to the American Rheumatism Association criteria (2), with a mean age of 65 years (range 53-73) and the mean duration of the disease of 26 years (range 15-50). Five H. pylori infected women without RA served as the control subjects (median age, 53 years; range, 48-56 years). All RA patients were treated with non-steroidal antiinflammatory drugs (NSAIDs) and corticosteroids. Gastric juice samples were taken with an endoscope between 08:00 and 12:00 after an overnight fast. Informed consent to participate in the study was obtained from all patients before enrollment. All RA patients in this study had macroscopic evidence of gastric ulcer or erosion as determined by the gastroduodenal endoscopy. The presence of the $H$. pylori infection was confirmed by the rapid urease test, serum antibody titre, or histological evidence of this bacterium. Among the RA patients, 3 were positive for $H$. pylori and 5 were negative. According to our previously described protocol (11), the five control subjects were assigned to receive amoxycillin and omeprazole with metronidazole. One month after completion of this eradication treatment, we confirmed the success of the $H$. pylori eradication in all the control patients and obtained gastric juice samples for evaluation of the mucin.

Extraction and isolation of mucin in gastric juice. The collected gastric juice was centrifuged at 1500 $\times g$ for $30 \mathrm{~min}$ at room temperature to remove the contaminating debris. The mucin in the gastric juice samples was extracted and isolated by a previously described method (4). Absolute ethanol $(6 \mathrm{~mL})$ was added to $2 \mathrm{~mL}$ of the supernatant obtained from the gastric juice to make a $75 \%$ ethanol concentration ( $\mathrm{v} /$ v). The resultant suspension was maintained at $4{ }^{\circ} \mathrm{C}$ overnight to complete the precipitation, after which the precipitate was collected by centrifugation ( 8000 $\times g$ for $30 \mathrm{~min}$ at $4^{\circ} \mathrm{C}$ ). The pellet was dissolved in distilled water $(2 \mathrm{~mL})$ and its hexose content was measured by the phenol-sulfuric acid method. The mucin content of the gastric juice was expressed as the hexose amount in the solution obtained by the precipitation method $(\mu \mathrm{g} / \mathrm{mL})$.

Fractionation of human gastric mucin. The obtained mucin solution was digested with $1 \mathrm{mg} / \mathrm{mL}$ chymotrypsin, extensively dialyzed against deionized water, lyophilized, and redissolved in $10 \mathrm{mM}$ Tris $\mathrm{HCl}$, $\mathrm{pH}$ 7.2. The sample was applied to a $5 \mathrm{~mm} \times 50 \mathrm{~mm}$ ion exchange column containing DEAE-Sepharose CL-6B (Pharmacia Fine Chemicals, Sweden) and eluted at $1 \mathrm{~mL} / \mathrm{min}$ with a discontinuous gradient concentration of $\mathrm{NaCl}(0,0.25,0.5,1.0 \mathrm{~mol} / \mathrm{L})$ in $10 \mathrm{mM}$ Tris $\mathrm{HCl}, \mathrm{pH}$ 7.2. The effluent solution collected from the DEAE-Sepharose column was checked by hexose measurement and the mucin recovery was found to be approximately $90 \%$.

Analysis of carbohydrate composition. The sugar composition of the fractionated mucin was determined using a Shimadzu GC7A gas chromatograph.

Statistical analysis. The differences in the mean values among the groups in the present study were analyzed by the one-way ANOVA with Dunett's test. P values less than 0.05 were considered to indicate statistical significance.

\section{RESULTS}

Fig. 1A shows a typical DEAE-Sepharose chromatography elution pattern of the mucins obtained from the gastric juice sample of a $H$. pylori-negative patient with RA. The gastric luminal mucins were divided into three fractions (Fr. I, Fr. II, and Fr. III) by ion exchange chromatography. These mucin Frs. I-III were further studied in order to determine whether they represented compositionally distinct mucin species using gas-liquid chromatography. The carbohydrate in Fr. III was composed of hexosamine, hexose, and sialic acid in the average ratio of $1.00: 1.24: 0.96$, expressed as moles per mole of hexosamine. In contrast, the proportion of sialic acid to each carbohydrate in Frs. I and II was much less than that in Fr. III (hexosamine: hexose: sialic acid $=1.00: 1.45: 0.00$ for Fr. I, $1.00: 1.32: 0.13$ for Fr. II). The mucin elution pattern of Fig. 1B is obtained from a $H$. pylori-positive patient with RA. The RA patients with $H$. pylori positive had higher contents of acidic mucin rich in sialic acid (Fr. III) when compared with the H. pylori negative RA patients. 


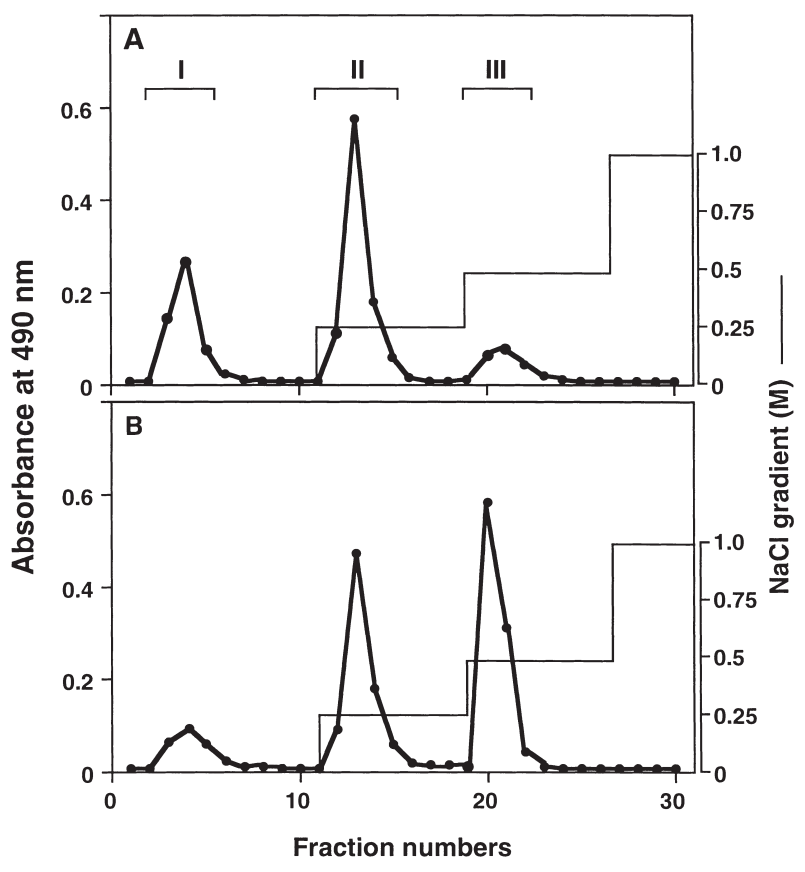

Fig. 1 DEAE-Sepharose column chromatography of mucins obtained from the gastric juice samples. (A) Chromatography elution pattern of an RA patient without $H$. pylori infection. (B) Elution pattern of an RA patient with $H$. pylori infection.

Since there was considerable variation in the mucin content in each fraction among individual patients, the percentages of acidic mucin fraction to the total gastric luminal mucin contents were calculated in the RA patients and control subjects. As shown in Fig. 2, the proportion of acidic mucin from the gastric juice of RA patients without $H$. pylori infection was significantly lower than those of RA with $H$. pylori or the control subjects. Fig. 2 also shows a decrease in the percentage of acidic mucin from the gastric juice of the control subjects after $H$. pylori eradication - a mean reduction of $22 \%$.

\section{DISCUSSION}

Gastric mucin is considered to be important for mucosal protection and is essential for the maintenance of the normal gastric function and resistance to diseases. However, our understanding of its clinical role in health and disease has been limited by the lack of detailed information on specific mucin compositional changes related to certain diseases. Mucin is a high molecular weight substance of which more than $70 \%$ is carbohydrates. The terminal region of the $O$-glycosidically linked carbohydrate chains is

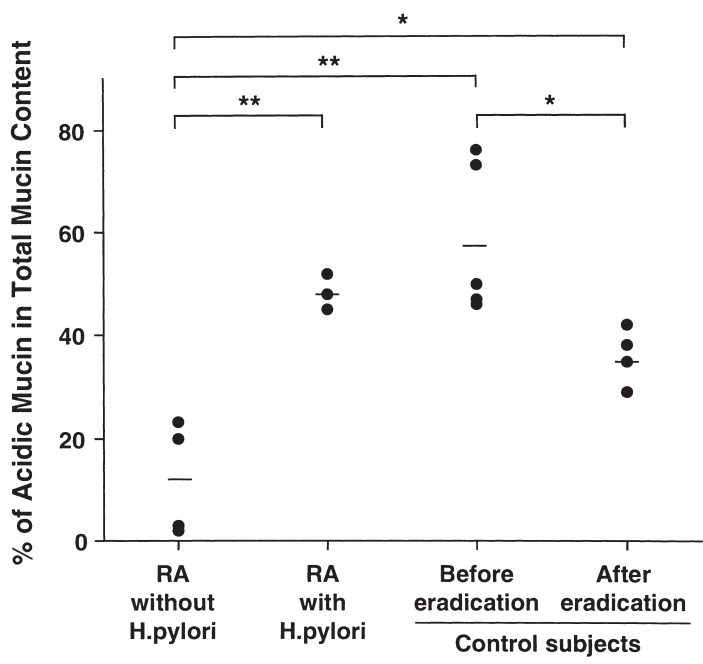

Fig. 2 Percentage of acidic mucin in total mucin content in 5 RA patients without $H$. pylori infection, 3 RA patients with $H$. pylori, and 5 control subjects before and after eradication treatment of the $H$. pylori infection. Horizontal line represents mean value of each group.

${ }^{* *} p<0.01,{ }^{*} p<0.05$.

comprised of sialic acid, hexose and hexosamine. We have been studying the biochemical and physiological properties of mucins in the rat stomach. Although the differences between humans and rats should be kept in mind, the results of our previous investigation indicated that mucin in human gastric juices may contain more than two distinct molecular species (5). The present study shows that the human gastric luminal mucins can be fractionated into three major components by a difference in the proportion of sialic acid to other carbohydrate components. According to the report by Podolsky and Isselbacher (13), the human colonic mucins were divided into six subclasses and a selective decrease in one colonic mucin subclass appeared to be associated with ulcerative colitis. Thus, it is conceivable that the alteration in certain fractions of the gastric luminal mucin contributes to systemic diseases.

In this study, only three among the 8 RA patients were positive for $H$. pylori. Our preliminary observation also indicated that there were $38 \mathrm{H}$. pyloripositive patients in $59 \mathrm{RA}$ cases (males/females $=4 / 55$; average age, 61; mean duration of the disease, 17 years). Asaka et al. (3) proved that the prevalence of $H$. pylori infection in Japan increased with age. For persons born before 1950, the percentage of $H$. pylori-positive persons was high (70-80\%) and relatively constant (3). Because certain anti-rheumatic gold compounds were reported to have an anti- $H$. pylori activity (8), we have checked whether RA pa- 
tients in the present study had received such gold compounds. Unfortunately, such records were not available. Our combined observations agree with those of previous investigations, which have found that patients with RA tended to have a low prevalence of $H$. pylori infection $(12,14)$.

The most striking finding in this study was that RA patients without $H$. pylori infection had lower percentages of acidic mucin, namely Fr. III having a markedly higher sialic acid content in the gastric juices than the other group subjects. Furthermore, our investigation provides compelling evidence of a selective decrease in the acidic mucin fraction from the gastric juice of the control subjects after H. pylori-eradication. It is possible that a high percentage of acidic mucin in the gastric juice may be related to an increased risk of $H$. pylori infection. Studies by several investigators have demonstrated that specific mucins affect $H$. pylori adherence to the gastric epithelium $(6,15,16)$. These mucins call for certain carbohydrate side-chains such as sialic acid and sulfate. Veerman et al. (16) reported that sulfated oligosaccharides on mucins provided receptor structures for attachment of $H$. pylori to the epithelial cells. Prior studies have indicated that $H$. pylori is able to bind to human sialomucin and that this binding prevents adherence of the organism to the gastric epithelium $(6,15)$. Although we have not examined the content of the sulfated mucin in the present experiment, past results $(6,15,16)$ and our findings show that the acidic mucin metabolism may have important implications for clarification of the intrinsic defense mechanism against $H$. pylori.

It is presently unclear why the acidic mucin content is low in RA patients, particularly in the non- $H$. pylori infected ones. However, our investigation raises the possibility that the gastric mucosae of RA patients have resistance against $H$. pylori infection. Recently, Kawakubo et al. (9) reported that the unique $O$-glycans in gastric mucin appears to function as a natural antibiotic, protecting the host from H. pylori. These findings suggest that the analyses of the certain mucin species are needed for the clinical trials from the viewpoint of gastric mucosal defense system. Further studies are now in progress to confirm these preliminary results, and in particular, the effect of NSAIDs and corticosteroids on the acidic mucin contents in human gastric juices.

In conclusion, the present results indicate that the analysis of the composition in the gastric luminal mucin may be a very useful tool for the evaluation of gastric homeostasis in RA patients.

\section{Acknowledgments}

Part of this work was supported by Grants-in-Aid for Scientific Research from the Japanese Ministry of Education, Science and Culture, and by the Integrative Research Program of the Graduate School of Medical Sciences, Kitasato University.

\section{REFERENCES}

1. Allen A, Hutton D, Leonard A, Pearson J and Sellers L (1986) The role of mucus in the protection of the gastroduodenal mucosa. Scand J Gastroenterol 21 (Suppl 125), 71-77.

2. Arnett FC, Edworthy SM, Bloch DA, McShane DJ, Fries JF, Cooper NS, Healey LA, Kaplan SR, Liang MH, Luthra HS, Medsger Jr TA, Mitchell DM, Neustadt DH, Pinals RS, Schaller JG, Sharp JT, Wilder RL and Hunder GG (1988) The American Rheumatism Association 1987 revised criteria for the classification of rheumatoid arthritis. Arthritis Rheum 31, 315-324.

3. Asaka M, Kimura T, Kudo M, Takeda H, Mitani S, Miyazaki T, Miki K and Graham DY (1992) Relationship of Helicobacter pylori to serum pepsinogens in an asymptomatic Japanese population. Gastroenterology 102, 760-766.

4. Azuumi Y, Ichikawa T, Ishihara K and Hotta K (1993) The validity of the ethanol precipitation method for the measurement of mucin content in human gastric juices and its possible relationship to gastroduodenal diseases. Clin Chim Acta 221, 219-225.

5. Goso Y, Ishihara K, Kurihara M, Sugaya T and Hotta K (1999) Rat gastric mucins recognized by monoclonal antibodies RGM21 and HIK1083: Isolation of mucin species characteristic of the surface and glandular mucosa. J Biochem 126, 375-381.

6. Hirmo S, Kelm S, Iwersen M, Hotta K, Goso Y, Ishihara K, Suguri T, Morita M, Wadstrom T and Schauer R (1998) Inhibition of Helicobacter pylori sialic acid-specific haemagglutination by human gastrointestinal mucins and milk glycoproteins. FEMS Immunol Med Microbiol 20, 275-281.

7. Ichikawa T, Koizumi W, Takeuchi H, Okayasu I, Hayashida H, Saigenji K and Ishihara K (2001) A new strategy for evaluation of the eradication treatment of Helicobacter pylori: validation of the analysis of mucin composition in human gastric juice. Clin Chim Acta 303, 69-73.

8. Janssen M, Dijkmans BA, Vandenbroucke JP, van Duijn W, Pena AS and Lamers CB (1992) Decreased level of antibodies against Helicobacter pylori in patients with rheumatoid arthritis receiving intramuscular gold. Ann Rheum Dis 51, 1036-1038.

9. Kawakubo M, Ito Y, Okimura Y, Kobayashi M, Sakura K, Kasama S, Fukuda MN, Fukuda M, Katsuyama T and Nakayama J (2004) Natural antibiotic function of a human gastric mucin against Helicobacter pylori infection. Science 305, 1003-1006.

10. Kinoshita Y, Kawanami C, Kishi K, Nakata H, Seino Y and Chiba T (1997) Helicobacter pylori independent chronological change in gastric acid secretion in the Japanese. Gut 41, $452-458$.

11. Koizumi W, Tanabe S, Hibi K, Imaizumi H, Ohida M, Okabe H, Saigenji K and Okayasu I (1998) A prospective randomized study of amoxycillin and omeprazole with and without metronidazole in the eradication treatment of Helico- 
bacter pylori. J Gastroenterol Hepatol 13, 301-304.

12. Mizokami Y, Tamura K, Fukuda Y, Yamamoto I and Shimoyama T (1994) Non-steroidal anti-inflammatory drugs associated with gastroduodenal injury and Helicobacter pylori. Eur J Gastroenterol Hepatol 6, S109-112

13. Podolsky DK and Isselbacher KJ (1983) Composition of human colonic mucin. Selective alteration in inflammatory bowel disease. J Clin Invest 72, 142-153.

14. Showji Y, Nozawa R, Sato K and Suzuki H (1996) Seroprev- alence of Helicobacter pylori infection in patients with connective tissue diseases. Microbiol Immunol 40, 499-503.

15. Tzouvelekis LS, Mentis AF, Makris AM, Spiliadis C, Blackwell C and Weir DM (1991) In vitro binding of Helicobacter pylori to human gastric mucin. Infect Immun 59, 4252-4254.

16. Veerman ECI, Bank CMC, Namavar F, Appelmelk BJ, Bolscher JGM and Nieuw Amerongen AV (1997) Sulfated glycans on oral mucin as receptors for Helicobacter pylori. Glycobiology 7, 737-743. 